\title{
PROPUESTA HACIA EL DEPORTE INCLUSIVO EN EDUCACIÓN SECUNDARIA OBLIGATORIA
}

\author{
María Jesús Lirola \\ Universidad de Almería \\ mariajesus.lirola@ual.es
}

Recepción Artículo: 15 abril 2020

Admisión Evaluación: 17 abril 2020

Informe Evaluador 1: 17 abril 2020

Informe Evaluador 2: 119 abril 2020

Aprobación Publicación: 20 abril 2020

\section{RESUMEN}

La propuesta de intervención que se realiza en este trabajo parte de la premisa del uso del deporte como herramienta inclusiva y socializadora. Es por ello que esta propuesta dirigida a la educación secundaria toma su foco de actuación dentro de la asignatura de Educación Física (EF). Son diferentes las investigaciones que muestran como contexto ideo aquel que engloba a la práctica de actividad física, debido a que el entorno de clase en EF favorece en mayor medida las relaciones sociales entre los alumnos/as que aquel en el que se imparten el resto de asignaturas y que por lo general representa un espacio cerrado con disposiciones inamovibles para los estudiantes. Por lo que se propone el trabajo en valores como la empatía, solidaridad, trabajo en equipo y apoyo social a través de una propuesta de intervención lúdica con actividades físicas. La intervención se realizará durante un periodo de 3 meses, en las clases de EF de una clase de $2^{\circ}$ de ESO, en la cual durante esa hora otro grupo de diversidad funcional entrará en dicha clase para realizar los juegos y actividades inclusivas que se propongan. Para dicha intervención se tendrán en cuenta aspectos metodológicos, sociales y contextuales. Como conclusión se puede decir que este tipo de propuestas se muestran necesarias para favorecer la inclusión real en la sociedad actual.

Palabras clave: inclusión social; educación secundaria; juego lúdico; deporte inclusivo

\section{ABSTRACT}

Proposal towards inclusive sport in compulsory secondary education. The proposal for intervention made in this work is based on the premise of the use of sport as an inclusive and socializing tool. That is why this proposal aimed at secondary education takes its focus of action within the subject of Physical Education (PE). Different research shows that the ideal context is that which encompasses the practice of physical activity, because the classroom environment in PE favours social relations between students to a greater extent than that in which the rest of the subjects are taught and which generally represents a closed space with immovable arrangements for students. Therefore, it is proposed to work on values such as empathy, solidarity, teamwork and social 


\section{PROPUESTA HACIA EL DEPORTE INCLUSIVO EN EDUCACIÓN SECUNDARIA OBLIGATORIA}

support through a proposal of recreational intervention with physical activities. The intervention will be carried out during a period of 3 months, in the PE classes of a 2nd year ESO class, in which during that hour another group of functional diversity will enter that class to carry out the games and inclusive activities that are proposed. For this intervention, methodological, social and contextual aspects will be taken into account. As a conclusion, it can be said that this type of proposals are necessary to favour real inclusion in today's society.

Keywords: social inclusión; secondary education; recreational play; inclusive sport

\section{INTRODUCCIÓN}

Este proyecto de intervención busca la mejora de la inclusión de las personas con diversidad funcional en el ámbito educativo, con el objetivo de la mejora y la puesta en práctica no solo de habilidades físicas sino también sociales. Para ello, se desarrollará una serie de intervenciones prácticas donde un grupo de personas con diversidad funcional y un grupo-clase que asiste a un centro de Educación Secundaria realizarán actividades conjuntas durante las horas de Educación Física que la propia clase tiene establecidas. De este modo se pretende buscar un trabajo inclusivo real donde se pongan en marcha el trabajo en valores aparte de los aspectos relacionados con la mejora de la condición física. Otro de los objetivos principales será mejorar las relaciones sociales entre individuos que poseen algún tipo de discapacidad y aquellos que se encuentran dentro de un marco normalizado. También, se trabajarán las relaciones intrapersonales, y con el entorno. Se trabajará a través del deporte empleando estrategias lúdicas y dinámicas para fomentar la movilidad, las habilidades sociales, el trabajo en equipo y la expresión corporal.

La palabra discapacidad se define como un gran número de diversas limitaciones funcionales que se estudian en todos los países del mundo. La discapacidad puede darse en diversas formas, ya que puede ser una deficiencia intelectual, física o sensorial, una enfermedad de carácter mental o física la cual necesite de atención médica continua. Este tipo de lesiones pueden ser a nivel transitorio o permanente (Hurtado, 2016). Según los estudios realizados recientemente por la Organización Mundial de la Salud (2011), en todo el mundo existen millones de personas que crecen con algún tipo de discapacidad, es decir, un $15 \%$ de las personas presentan algún tipo de condición. En América Latina se ha podido comprobar que alrededor de un 10\% de la población (85 millones) presenta algún tipo de discapacidad.

El deporte orientado a personas con diversidad funcional se denomina deporte adaptado. Este tipo de deporte presenta una serie de rasgos específicos como son por ejemplo el contexto, las funciones, la manera de intervenir, las cuales son diferentes en relación a otras modalidades. El deporte adaptado presenta ciertas características, entre las que se pueden destacar: usuarios que presentan diferente motricidad, reglas adaptadas, adecuación a determinadas edades evolutivas, etc. (Vázquez, 2000). El deporte adaptado tiene una serie de funciones como son: sensibilizar a la población en general sobre los inconvenientes que presentan las personas con diversidad funcional y sobra la práctica deportiva de manera particular, utilizar el deporte adaptado como un medio para acercar la diversidad al deporte, para la normalización de las personas con diversidad funcional con el objetivo de derribar barreras y así conseguir favorecer programas individualizados para grupos o personas con características específicas, consiguiendo así, proporcionar más formas de trabajo, y por último realizar programas físicos adaptados creando mayores perspectivas no solo de carácter teórico, sino de manera práctica para su posterior aplicación (Vázquez, 2000).

El ámbito y contextualización del deporte ha ido experimentando cambios de manera progresiva con el paso del tiempo, desde aquellos tiempos donde la gimnasia sueca, aquella tan popular en los años 60 donde los alumnos reproducían los diferentes movimientos que les proponía el profesor, hasta la actualidad donde el deporte es de carácter más individualizado con el objetivo de proporcionar a cada alumno y alumna lo que necesita (Sazatornil Pinedo, 2013). Cada persona en su propia naturaleza es diferente a las demás, lo cual proporciona a cada individuo una esencia y características únicas. En ello, nace el sentido de la idea de educar en diversidad. Idea que será el eje central de este proyecto a la hora de hablar sobre la inclusión de personas con discapacidad funcional en materia de educación teniendo como base el deporte. Según Sazatornil Pinedo (2013), el deporte es 
un medio muy útil para romper barreras en diferentes campos de intervención y así conseguir una integración óptima. Según los autores Levoratti y Zambaglione (2015), el deporte social ha creado un nuevo concepto de las actividades físicas, deportivas y recreativas en las que se incluyen a toda la comunidad sin excluir a nadie por edad, sexo, diversidad funcional, cultura, etnia o raza. Esto genera lugares y espacios para la enseñanza de valores y hábitos que podemos incluir en la enseñanza de niños, niñas, adultos, ancianos, etc., basado en un proyecto global de inclusión social. Como señala Rivas y Vaíllo (2013), el deporte actúa sí o sí como una herramienta de inclusión, por ello es necesario que todos los profesionales que se dediquen a la enseñanza, tanto formal como informal, actúen en esta línea y llegar a los sectores más excluidos.

La diferencia y ventaja que presenta el deporte, según Rivera (2014), es que se trata de una herramienta de carácter informal, bastante atrayente entre el sector joven, con una gran capacidad de enseñar valores y poder ponerlos en práctica de manera divertida y participativa. La misma autora señala que el deporte no solo enseña valores que se lleven a cabo durante su práctica, sino que podemos trasladarlos a nuestra vida en general como son la cooperación, el respeto, la honestidad, etc., necesarios para crear una cohesión social que perdure en el tiempo. Igualmente, ésta señala que los programas diseñados con deporte pueden conseguir una inclusión total, sobre todo en áreas de exclusión social o con personas con capacidades diferentes.

\section{OBJETIVOS}

El presente proyecto se basa en el empleo del juego como principal herramienta educativa, ya que facilita el aprendizaje y desarrollo cognitivo, afectivo, personal y social, entre otros. Además, como afirma Del Toro Alonso (2013), en relación al área motora, el juego posibilita exploración, movimiento y manipulación del entorno, y favorece la coordinación dinámica general. Asimismo, el uso del juego permite manejar unas normas comunes de grupo que favorecen la inclusión social de los y las participantes. En cuanto al colectivo de personas con discapacidad, estas suelen presentar características, como baja autoestima, falta de autoconcepto o poca tolerancia a la frustración, que afectan de forma negativa en su desarrollo y aprendizaje, por lo que el juego es una gran herramienta para mejorar su situación, ya que facilitan la mejora de dichos factores psicológicos y, en consecuencia, de su calidad de vida.

\section{MÉTODO}

\section{Participantes}

Este programa de intervención va dirigido a personas que presenten diversidades funcionales y/0 cognitivas, también a personas que se encuentren dentro de un marco normalizado. Dichos usuarios tendrán edades comprendidas entre los 13 y 16 años de edad, siendo el tamaño de este grupo de 7 participantes. Además, este proyecto se llevará a cabo dentro de un contexto educativo, se trabajará con los alumnos/as de una clase de $2^{\circ}$ de Educación Secundaria Obligatoria $(N=24)$ de un Centro de la Provincia de Almería.

\section{Planificación}

La intervención tendrá lugar durante el periodo de un trimestre escolar (i.e., tres meses), interviniendo 2 días en semana, un total de 1 hora en cada sesión. De esta manera el programa inclusivo contará con un total de 25 sesiones.

\section{Procedimiento}

El proyecto se debe fundamentar en una serie de principios, los cuales se tendrán en cuenta a la hora de poner en marcha la intervención:

- El ambiente ha de posibilitar el conocimiento y acercamiento de todas las personas del grupo. Se debe favorecer la cohesión y el logro de los objetivos comunes.

- Todos y todas deben tener contacto con materiales y actividades diversas que les permitan adquirir un amplio abanico de aprendizajes cognitivos, sociales y afectivos. 


\section{PROPUESTA HACIA EL DEPORTE INCLUSIVO EN EDUCACIÓN SECUNDARIA OBLIGATORIA}

- Dentro de una educación inclusiva es enriquecedor ofrecer escenarios distintos.

- El entorno ha de ser construido activamente por todos los participantes, viéndose en él reflejadas sus peculiaridades y su propia identidad.

Durante el desarrollo del proyecto se deben crear las condiciones idóneas, que transmitan seguridad y bienestar físico y emocional, que faciliten la aceptación mutua y un ambiente de confianza, el reconocimiento de la identidad, etc. Se deben emplear estrategias educativas que promuevan situaciones de aprendizaje que estimulen el desarrollo de las competencias deseadas y las metas que se quieran alcanzar. Concretamente, en este proyecto algunas de las estrategias educativas a usar serán el empleo de actividades lúdico-deportivas, el aprendizaje colaborativo, simulaciones, etc.

Por otro lado, un correcto ambiente de aprendizaje se divide en cuatro dimensiones, los cuales se tendrán en consideración durante la práctica (Cabrera Albert y Fariñas León, 2019):

- Dimensión física: Lo relacionado con el aspecto material del ambiente y su organización.

- Dimensión funcional: la manera en la que se utilizan los espacios para la realización de las actividades y sus condiciones.

- Dimensión temporal: Se tendrá en cuenta la organización del tiempo y cuándo será utilizado cada espacio.

- Dimensión relacional: las diversas relaciones interpersonales que se logran establecer durante el desarrollo del proyecto de intervención.

\section{RESULTADOS ESPERADOS}

\section{Competencias y expectativas a desarrollar}

Como se ha expuesto en los apartados anteriores, el presente proyecto va enfocado a la inclusión de la diversidad. Para ello, se trabajarán una serie de destrezas relacionadas con la comunicación, las relaciones sociales, habilidades sociales, etc. A continuación, se exponen las competencias a desarrollar:

- El uso de los sentidos (tacto, olfato y oído) para reconocer y conocer.

- La posibilidad de desarrollar estrategias para comunicarnos con el otro a través de espacio.

- El autocontrol de los movimientos y conciencia de los impulsos.

- La interacción desde el respeto y la aceptación del otro.

- El cuerpo, de pies a cabeza como lenguaje que posibilita la comunicación para descifrar y aprender.

- Esperamos que nuestros usuarios sean capaces de experimentar un medio sin coacciones en el que podrán desarrollar los ejercicios sin ataduras.

- Tomar consciencia de su cuerpo, que experimenten con el mismo, conozcan sus limitaciones y sus posibilidades.

- El espacio como medio para respetar nuestras individualidades, pero también para compartir sensaciones y percepciones de la realidad en la que vivimos.

\section{Ambiente de aprendizaje}

Se pretende conseguir un ambiente inclusivo, dinámico, donde los valores de respeto, empatía, cooperación y ayuda mutua estén presentes en todo momento, y donde todos los y las participantes creen unas redes de confianza y amistad, con la intención de construir lazos entre ellos/as de modo que amplíen su red de apoyo y su grupo de iguales. Por ello, se intentará que en todo momento se establezca un ambiente de aprendizaje adecuado para propiciar la comunicación entre todos/as, además de concretar unas normas básicas de comportamiento y unas reglas específicas de cada juego.

Durante el desarrollo de las sesiones se van a considerar dos aspectos fundamentales para un correcto ambiente y proceso de enseñanza-aprendizaje (Aguirre et al., 2007). El primero es la existencia de un espacio de información, donde el profesor/a pueda transmitir de forma clara los conocimientos necesarios al grupo, como la explicación del juego y sus reglas, para el correcto desarrollo de las actividades que se lleven a cabo. Por otro 
lado, será importante que las personas implicadas en este proyecto ya sea el profesorado del centro o los monitores/as del grupo de diversidad funcional propicien un espacio de interacción, en el cual favorezcan la creación de relaciones interpersonales entre todos y cada uno de los individuos presentes. Asimismo, según Rodríguez Vite (s.f.), para la creación de dicho ambiente de aprendizaje se tendrán en cuenta los siguientes elementos:

- Organización espacial: Un espacio amplio, con una buena iluminación y ventilación, etc. y, además, una correcta distribución del espacio y materiales.

- Disposición de los materiales necesarios y el buen estado de los mismos.

- La organización debe ser la más adecuada posible para alcanzar los objetivos y, en este caso, teniendo en cuenta aspectos más especiales como las características de los y las participantes.

\section{DISCUSIÓN/CONCLUSIONES}

Los problemas a los que se enfrentan las personas con dificultades motoras, cognitivas o con cualquier otra diversidad funcional son bastantes, en este punto cabe destacar que una de las mayores complejidades es al acceso y disfrute de la información, en él quedan excluidos debido a un sistema socioeducativo que no tiene en cuenta las realidades de otros usuarios. Como indican Lafuente, Ballesteros y Filippi (2018), el problema no está en las características de los usuarios sino en los ámbitos que lo rodean, los contextos educativos no consiguen la plena integración y suponen más barreras que ventajas puedan ofrecer. La información y el conocimiento es derecho de todos, tal y como exponen Lafuente, Ballesteros y Filippi (2018) tienen la misma necesidad de información que el resto, por ello el sistema educativo debe garantizar un espacio donde se puedan realizar personalmente, tomen decisiones y se desarrollen de manera autónoma e independiente, para alcanzar una igualdad social y educativa.

Los usuarios son heterogéneos, lo cual demanda que los sistemas educativos estén al nivel y se ajusten a las individualidades tales como las adaptaciones curriculares pertinentes para conseguir la inclusividad de los individuos y a la vez conseguir una educación inclusiva. Se trata, como indican Sánchez, Romero y Padrón (2019), de una reestructuración que responda y acoja a la diversidad independientemente de sus características individuales, que a su vez son enriquecedoras, abordando problemas que los engloban desde los más distantes hasta los cercanos y cotidianos.

El problema en el desarrollo de las escuelas ocurre a su vez lejos de las mismas, en dimensiones donde es necesario un cambio firme que produzca procesos de transformación en la educación, mencionando pues a la necesidad de culturas, políticas y prácticas inclusivas (Sánchez, Romero y Padrón, 2019), es decir, una comunidad que valore la diversidad, que garantice su participación e igualdad y que, desde la implicación y reflexión desarrolle prácticas que incidan sobre la educación y el aprendizaje.

Según Sánchez (2018) existen aún muchos errores en el sistema educativo, ya sea por las metodologías utilizadas o por los enfoques pedagógicos, para los cuales se han necesitado cambios. El conocimiento en general es diverso y su forma de transmisión debe de ser de este modo también diversificada, para no caer en el error de continuar con formatos de clases homogéneas que no dan pie a la creatividad ni al cambio. El formato de la educación en todas sus esferas se ha ido desarrollando, pero también es necesario que las propuestas educativas se desarrollen con nuevos puntos de vista y con toque innovadores, para de esta manera dar solución y respuesta a muchos problemas que dificultan la normalidad en los centros educativos normalizados y en aquellos de usuarios con diversidad. Decisiones como la adaptación curricular es una respuesta a la diversidad, un elemento determinante para garantizar un currículo flexible (Sánchez, Romero y Padrón, 2019), dispuesto a la apertura y no a la rigidez que conocen los sistemas educativos que según la ley la perspectiva se orienta a responder a demandas externas económicas y políticas en ambos casos impersonales desatendiendo a la heterogeneidad que se menciona, una injusticia social que corresponde a las concepciones liberales de nuestra sociedad.

Las adaptaciones curriculares son destacables por su accesibilidad e individualidad, para garantizar su verdadera implementación han de estar en proyectos institucionales, centrándose en el presente y en la visión de futuro de los individuos, con una programación a priori, con un trabajo en red, en colaboración, es decir, instan- 


\section{PROPUESTA HACIA EL DEPORTE INCLUSIVO EN EDUCACIÓN SECUNDARIA OBLIGATORIA}

cias que no trabajan de manera separada, sino que se coordinan y cooperan, un trabajo interdisciplinar que realiza ejercicios de seguimiento (Sánchez, Romero y Padrón, 2019). Esto consiste en dar un paso hacia la inclusividad, un reto a día de hoy para la educación, un trabajo arduo que necesita de la coexistencia de diferentes instancias que apoyen y trabajen a la par con el sistema educativo para hacer de este un lugar donde no haya barreras para el alumnado con diversidad funcional, sin caer en la separación del alumnado de su grupo de pares provocando su exclusión.

Hay autores como Aquino Zúñiga, García Martínez e Izquierdo (2012) que a su vez añaden la evaluación de la calidad y eficacia de las escuelas con el matiz de involucrar a los actores de la comunidad, usuarios activos, que a partir de la reflexión pueden interferir disminuyendo las barreras, analizar las posturas del sistema educativo posibilitando la participación de los estudiantes, haciendo de esta una comunidad más inclusiva debido a prácticas que incluyen a diversidad de agentes en el cambio. Una escuela abierta y en contacto con la comunidad retroalimenta la capacidad de superar un currículo fijo, haciéndolo más en contacto con la sociedad para paliar las necesidades a las que hay responder del alumnado con diversidad.

Las adaptaciones deben a su vez superar los problemas que pueden darse en la escuela ya sean de inmobiliaria, iluminación, sonido, suprimiendo, adaptando, moldeando y desarrollando material atractivo, diverso e integrador, que siendo usuarios con diversidad funcional y/o cognitivo o no, será positivo. Sin embargo, la continua visión negativa de la sociedad y su incidencia en la escuela se ha preservado a la separación y exclusión que a replantear nuevas metodologías. Por ello, como indican Sánchez, Romero y Padrón (2019) el docente tiene un gran papel de mediador para aportar la parte reflexiva de todas estas visiones, todos los medios que garanticen interacciones, aprovechar la diversidad para enriquecer. Sin embargo, aunque los docentes como dice Sánchez (2018) estén de acuerdo, ven afectado el funcionamiento y la demanda de recursos les hace creer que la labor no va como debe, unido todo a ello al sentimiento de compasión. Salta a la vista que son problemas que van a suponer barreras a la hora de trabajar con la diversidad, por ello, son necesarios docentes con predisposición y con formación suficiente para una inclusividad real en el sistema educativo.

Por ello, la creatividad dentro de un sistema educativo abierto posibilitará la resolución de obstáculos, ya que le permitirá al profesorado reformular los conocimientos y creencias preestablecidas, construyendo nuevas estructuras para relacionar experiencias, elaborar ideas y llevarlas a la hora de la práctica dando lugar a una posible mejora (Llamazares, Arias y Melcon, 2019), porque una vez en contacto con nuevas proyecciones y razonamientos la visión hacia la realidad que se les presenta puede ser cambiada.

La capacidad de aprendizaje y desarrollo son inherentes a las características de los seres humanos, la clave está en los oportunos procesos y técnicas que obtengan el máximo provecho dentro del contexto educativo, de la diversidad que en el convive y de un uso de los recursos de una forma viable y accesible para todos los individuos. Según García (2011) debido a la condición de las personas con diversidad la representación mental suele ser más débil, por lo que para ellos el conocimiento de la realidad será diferente. Por lo tanto, realizar propuestas dentro del contexto del deporte, es sin lugar a duda un medio donde se rompe con las barreras para ofrecer un espacio que posibilita a la diversidad dominar su esquema corporal, el acceso a sentir y percibir la realidad que les engloba, identificarse dentro de la misma y también conocer la posiciones de los otros, dando lugar a la comunicación con el resto de iguales (Martín, 2019). Por lo que es un medido didáctico creativo e inclusivo que enfocándolo de la forma más idónea responderá a las problemáticas educativas, y a la adaptación del sistema educativo 0 a parte de este a la heterogeneidad de individuos.

La intervención, por tanto, con personas que han sufrido esa exclusión, o que están en riesgo de sufrirla es imprescindible. Por ello esta propuesta de intervención socioeducativa se centrará en la intervención con un grupo de estudiantes de $2^{0}$ de secundaria junto con un grupo de siete participantes de entre los 12 y 16 años que tengan cualquier tipo de diversidad funcional. El lugar de actuación será en la pista deportiva de un Centro de Educación Secundaria de la provincia de Almería. El trabajo con este grupo se centrará principalmente en:

- Lograr una empatía real dentro de todo el grupo. 
- Reconocer que todos/as presentamos más dificultades en algunas habilidades que en otras.

- Empoderar a todos los participantes para que se puedan desenvolver con mayor facilidad en su día a día, dotándolos de competencias necesarias para ello.

- Ofrecerles la oportunidad de experimentar en primera persona la inclusión que existe fuera de un centro educativo, en la sociedad vivimos todos juntos y no estamos agrupados por edad, capacidades o gustos personales, de este modo si aprendemos de verdad a convivir y ofrecer una ayuda mutua haremos del mundo un lugar mejor.

- Trabajar en valores y la inclusión real.

Como conclusión a todo lo visto anteriormente, en la actualidad se debe abogar por nuevas formas educativas, más creativas y diferentes, incluyendo métodos, actividades creativas y artísticas, es decir, adaptándose al alumnado con dinámicas que motiven a desarrollar conceptos y habilidades, el trabajo de conocimientos y expresiones, favoreciendo el aprendizaje multisensorial (Llamazares, Arias y Melcon, 2019). Salirse de los esquemas de aprendizaje que ocasionan dificultades a fórmulas creativas que den lugar a un ambiente de expresión, motivación, creación e innovación, lo cual eliminará barreras a usuarios con diversidad para mejorar la autoestima, autoconcepto y donde trabajen la comunicación. Está claro que una escuela inclusiva va de la mano de procesos creativos para retar las complejidades que plantea el sistema educativo tradicional.

\section{REFERENCIAS BIBLIOGRÁFICAS}

Aguirre, P., Gil, J. M., González, J. L., Osuna, V., Polo, D. C., Vallejo de Castro, D., \& Prieto, I. (2007). Manual de atención al alumnado con necesidades específicas de apoyo educativo derivadas de discapacidad visual y sordoceguera. Andalucía, España: Consejería de Educación, Junta de Andalucía. Recuperado de http://www. juntadeandalucia. es/educacion/nav/contenido. jsp

Aquino Zúñiga, S., García Martínez, V., e Izquierdo, J. (2012). La inclusión educativa de ciegos y baja visión en el nivel superior: Un estudio de caso. Sinéctica, 39(1), pp.1-21.

Cabrera Albert, J. S. y Fariñas León, G. (2019). El estudio de los estilos de aprendizaje desde una perspectiva vigostkiana: una aproximación conceptual. Revista Iberoamericana de Educación. Recuperado de http://www.rieoei.org/deloslectores/1090Cabrera.pdf

Del Toro Alonso, V. (2013). El Juego como herramienta educativa del Educador Social en actividades de Animación Sociocultural y de Ocio y Tiempo libre con niños con Discapacidad. RES Revista de Educación Social, (16), 1-13.

García, M. (2011). El niño con discapacidad visual. ¿Cómo se desarrolla en el niño ciego la representación, interacción y función simbólica? Extraído de http://www.eduinnova.es/monografias2011/feb2011/visual.pdf

Hurtado, L. T. (2016). Inclusión educativa de las personas con discapacidad en Colombia. CES Movimiento y Salud, 45-55

Lafuente, G., Ballesteros, C. y Filippi, J. (2018). Desarrollo de una plataforma de capacitación a distancia utilizable y abierta para personas con discapacidad visual. [online] Sedici.unlp.edu.ar.

Levoratti, A. y Zambaglione, D. (2015). La recreación y el deporte social como medio de inclusión. Berazategui: Buenos Aires: Engranajes de la cultura.

Llamazares, J., Arias, A. y Melcon, M. (2019). Revisión teórica de la discapacidad visual, el papel fundamental de la unión entre la creatividad y la educación. Journal on Innovation and Sustainability, 10(1), pp.86-100.

Martín, P. (2019). Alumnos con discapacidad visual. Necesidades y respuesta educativa. [online] Asociaciondiwork.org. Available at: http://www.asociaciondiwork.org/Documentacion/Diversidad_funcional/Normal/Educacion/Necesidades\%20y\%20respuesta\%20educativa.pdf [Accessed 10 Nov. 2019].

Organización Mundial de la Salud, (2011). Informe mundial sobre la discapacidad. Ginebra, Suiza.

Rivas, D. S., \& Vaíllo, R. R. (2013). Actividades físicas y deportes adaptados para personas con discapacidad. Paidotribo.

Rivera, A.E. (2014). El deporte como herramienta para la construcción de una paz sostenible (Tesis Universitaria). 


\section{PROPUESTA HACIA EL DEPORTE INCLUSIVO EN EDUCACIÓN SECUNDARIA OBLIGATORIA}

Universidad del Azuay, Cuenca, Educador.

Rodríguez Vite, H. (s.f.). Ambientes de Aprendizaje. Recuperado de https://www.uaeh.edu.mx/scige/boletin/huejutla/n4/e1.html\#refe0

Sánchez, D., Romero, R. y Padrón, J. (2019). Inclusión de personas con discapacidades auditivas y visuales en la investigación. Revista de Estudios Interdisciplinarios en Ciencias Sociales, 21(1), pp.221-241.

Sánchez, J. (2018). Experiencia de un alumno con discapacidad visual en el sistema educativo español. Revista Nacional e Internacional de Educación Inclusiva, 11(2), pp.117-138.

Sazatornil Pinedo, P. (2013). La integración del alumnado con discapacidad visual en Secundaria a través de la Educación Física. Una propuesta de taller de sensibilización hacia la ceguera. (Trabajo Fin de Master). Universidad de Cantabria: Cantabria, España.

Vázquez, F. J. H. (2000). El deporte para atender a la diversidad: deporte adaptado y deporte inclusivo. Apunts. Educación física y deportes, 2(60), 46-53. 